\title{
Antimicrobials administration time in patients with suspected sepsis: is faster better? An analysis by propensity score
}

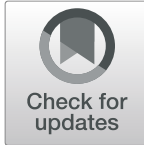

Johana Ascuntar ${ }^{1}$, Deibie Mendoza ${ }^{2}$ and Fabián Jaimes ${ }^{1,3,4^{*}}$

\begin{abstract}
Background: Early use of antimicrobials is a critical intervention in the treatment of patients with sepsis. The exact time of initiation is controversial and its early administration may be a difficult task in crowded emergency departments (ED). The aim of this study was to estimate, using a matched propensity score, the effect on hospital mortality of administration of antimicrobials within 1 or 3 hours, in patients admitted to the ED with sepsis.

Methods: This was a secondary analysis of a multicenter prospective cohort. Patients included in the study were older than 18 years, hospitalized between 2014 and 2016 with suspected sepsis, and admitted to ED of three tertiary care university hospitals in Medellín, Colombia. A propensity score analysis for administration of antimicrobials, both within 1 and $3 \mathrm{~h}$ of admission by the ED, was fitted with 28 variables related with clinical attention and physiological changes. As a sensitivity analysis, a logistic regression model was fitted for antimicrobial use adjusted both by propensity score and confounding variables.

Results: The study cohort was composed of 2454 patients with a median age of 62 years (IQR $=46-74)$. Among them, 32\% $(n=781)$ received antibiotics within $3 \mathrm{~h}$ and $14 \%(n=340)$ within the first hour. The main diagnoses were urinary tract infection (28\%, $n=682)$ and pneumonia $(27 \%, n=671)$. Blood cultures were obtained in $87 \%$ ( $n$ $=2140)$ and yielded positive in 29\% ( $n=629)$, mainly with Escherichia coli $(37 \%, n=230)$, Staphylococcus aureus $(21 \%, n=132)$, and Klebsiella pneumoniae $(10.2 \%, n=64)$. The hospital mortality rate was $11.5 \%(n=283)$. There were no significant differences in mortality, after adjustment, using antimicrobials either in the first hour (OR 1.03; $95 \% \mathrm{Cl}=0.63 ; 1.70)$ or $3 \mathrm{~h}(\mathrm{OR} 0.85 ; 95 \% \mathrm{Cl}=0.61 ; 1.20)$. There were no changes with different models for sensitivity analysis.
\end{abstract}

Conclusions: Despite the obvious constraints given for sample size and residual confounding, our results suggest that we need a more comprehensive approach to sepsis and its treatment, considering early detection, multiple interventions, and goals beyond the simple time-to-antimicrobials.

Keywords: Sepsis, antimicrobials, antibiotics, propensity score, in-hospital mortality

\footnotetext{
*Correspondence: fabian.jaimes@udea.edu.co

'GRAEPIC—Clinical Epidemiology Academic Group (Grupo Académico de Epidemiología Clínica), the University of Antioquia, Medellín, Colombia

${ }^{3}$ Department of Internal Medicine, University of Antioquia, Medellín, Colombia

Full list of author information is available at the end of the article
}

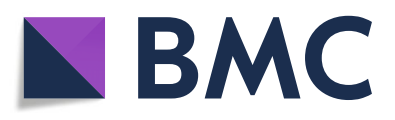

(- The Author(s). 2020 Open Access This article is licensed under a Creative Commons Attribution 4.0 International License, which permits use, sharing, adaptation, distribution and reproduction in any medium or format, as long as you give appropriate credit to the original author(s) and the source, provide a link to the Creative Commons licence, and indicate if changes were made. The images or other third party material in this article are included in the article's Creative Commons licence, unless indicated otherwise in a credit line to the material. If material is not included in the article's Creative Commons licence and your intended use is not permitted by statutory regulation or exceeds the permitted use, you will need to obtain permission directly from the copyright holder. To view a copy of this licence, visit http://creativecommons.org/licenses/by/4.0/. The Creative Commons Public Domain Dedication waiver (http://creativecommons.org/publicdomain/zero/1.0/) applies to the data made available in this article, unless otherwise stated in a credit line to the data. 


\section{Background}

In recent years, sepsis has been established as a public health problem with up to 30 million cases per year in the world and approximately five million deaths estimated, although with predominant information from high-income countries [1]. The Surviving Sepsis Campaign has established several pillars in the management of patients with sepsis [2]. Among the guidelines included are the initial resuscitation with intravenous fluids, the obtaining of blood cultures, the measurement of lactate levels, the control of the source of infection, antimicrobial therapy, and the use of vasopressors.

Although compliance with these elements has been associated with a reduction in mortality in patients with sepsis [3], their general implementation has not been without controversy $[4,5]$. Particularly, the early administration of antimicrobials in a real-life setting ED presents, among others, logistical difficulties. Controversies persist regarding the optimal time to start antimicrobials (within $1 \mathrm{~h}, 3 \mathrm{~h}$, or some other time point) and the correct definition of time zero (either after admission to the ED or after recognition of sepsis) [6]; this is because its recognition can be difficult and its specific onset is rarely known. With this in mind, it is important to consider that the indiscriminate use of antimicrobials can favor resistance and the occurrence of adverse effects, this being a potential disadvantage of the early administration of antimicrobials, together with higher inappropriate prescription rates [7].

A study in this field is necessary because the evidence of time-to-antimicrobials that supports the most recent guidelines comes mainly from retrospective studies, due to the ethical and logistical considerations of a clinical trial in the area. Having in mind that some other prospective studies controvert these 1-h guidelines, this investigation seeks to estimate, through the matching of the propensity score [8], a statistical technique that favors the reduction of bias due to prognostic factors [9], the effect of administering antimicrobials within 1 or $3 \mathrm{~h}$ on in-hospital mortality of patients admitted to ED with sepsis.

\section{Materials and methods}

\section{Study and design}

Secondary analysis obtained from a prospective cohort study [10]. The purpose of our primary study was to estimate the effect of each component of early goaldirected therapy (EGDT) protocol, as well as the effect of antibiotics use, on in-hospital mortality of patients with septic shock (according to the Sepsis-2 definition). The study was conducted in emergency departments and critical care units of three tertiary care university hospitals in the city of Medellín, Colombia: the Hospital Universitario San Vicente Fundación (HUSVF, 560 adult beds and 45 ICU beds in 4 units), the Hospital Pablo Tobón Uribe (HPTU, 360 adult beds and 40 ICU beds in 3 units), and the IPS Universitaria León XIII (IPSU, 450 adult beds and 24 ICU beds in 2 units). The period of patient recruitment and data collection was between June 2014 and February 2016 [10], and for the current study, we analyzed the total eligible population with any organ dysfunction, in addition to those with septic shock.

\section{Study population}

Inclusion criteria are as follows: patients aged greater than or equal to 18 years old; admitted to the ED with suspected sepsis, septic shock, or a record in the clinical history of infection under the criteria of the Center for Disease Control and Prevention (CDC) [11]; and at least one of the following criteria for organ dysfunction: Glasgow scale $<15 ; \mathrm{PaO}_{2} / \mathrm{FiO}_{2}$ index $<300$ or the need for mechanical ventilation; urinary output $<0.5 \mathrm{ml} / \mathrm{kg} / \mathrm{h}$ for $2 \mathrm{~h}$ reported in clinical record; creatinine $(\mathrm{CR})>2 \mathrm{mg} / \mathrm{dL}$ with no history of previous kidney disease or an $0.5 \mathrm{mg} /$ dl increase over previous values; international normalized ratio (INR) $>1.5 \mathrm{~s}$; partial thromboplastin time $($ PTT $)>60 \mathrm{~s}$; platelet count $(\mathrm{PLT})<150,000$ cells $/ \mathrm{mm}^{3}$; total bilirubin ( $\mathrm{T}$ Bil) $>2 \mathrm{mg} / \mathrm{dl}$; lactic acid (LA) $>2$ $\mathrm{mmol} / \mathrm{l}$; capillary refill time more than $2 \mathrm{~s}$; systolic blood pressure $(\mathrm{SBP})<90 \mathrm{mmHg}$ or mean arterial pressure (MAP) $<70 \mathrm{mmHg}$ during the first $6 \mathrm{~h}$ after admission. The current Sepsis-3 criteria were not part of the study population collection process because these were published in late February 2016. However, we analyzed the total eligible population with any organ dysfunction, which is overly similar to the current one based on the SOFA score.

Exclusion criteria are as follows: refusal by the patient, his family, or the attending physician to participate in the study; concurrent diagnoses of pregnancy, myocardial infarction, stroke, asthmatic crisis, arrhythmia, trauma, gastrointestinal bleeding, seizure not due to meningitis, psychoactive substance overdose, surgery < $24 \mathrm{~h}$, burns, CD4 count $<50$ cells $/ \mathrm{mm}^{3}$, hyperosmolar status, diabetic ketoacidosis or cirrhosis; discharge or remission in the first $24 \mathrm{~h}$ of hospitalization; prior participation in the study; referral from another institution where they had been hospitalized for more than $24 \mathrm{~h}$ or a no-resuscitation order.

\section{Measurements}

\section{Exposure variables}

The procedures and treatments performed during the first $24 \mathrm{~h}$ of hospital stay were recorded, according to the original protocol of Rivers et al. [12]. The administration of antimicrobials was recorded during the first $24 \mathrm{~h}$ of hospital stay (antimicrobial administration within 
$1 \mathrm{~h}$ and within $3 \mathrm{~h}$; time zero was defined as admission to the ED, which we consider more relevant from the clinical point of view). The type and dosage of the treatments were taken into consideration: intravenous fluids, antimicrobials, vasopressors, and transfusions. In addition, the researchers evaluated the antimicrobial scheme administered by classifying it as adequate or inadequate, according to the final microbiological isolation or, if this does not exist, according to the clinical criteria in each case.

\section{Potential confounding variables}

As potential confounding variables were taken into account: age, quantity of intravenous fluids (IVF), blood cultures in the first $3 \mathrm{~h}$, and Charlson Index [13]; the severity of sepsis, assessed using Sepsis-related Organ Failure Assessment (SOFA) score [14] and Acute Physiology and Chronic Health Evaluation (APACHE II) scoring system [15]; and the confirmed diagnosis of infection, the inadequate use of antimicrobials, and the lactate values at admission. The definition of sources of infection was standardized according to the CDC criteria [11]. In the included hospitals, the treatment strategy was mainly determined by the treating physician. Nevertheless, all the institutions had availability of the resources needed to apply the complete treatment and also had a basic suggested protocol that included continuous monitoring, IVF bolus with crystalloid according to the blood pressure and central venous pressure, early administration of adequate antimicrobials in the first $3 \mathrm{~h}$ depending on the suspected focus of infection, taking of appropriate cultures and initiation of a vasopressor when blood pressure did not improve with IVF.

\section{Primary outcome}

In-hospital mortality

\section{Data source}

In the primary study, all patients who presented to the ED with a diagnosis of infection, sepsis, severe sepsis, or septic shock were screened. The source of the infection and the presence of organ dysfunction or shock were verified with the data extracted from the clinical records in the first $6 \mathrm{~h}$. All data on the diagnosis and treatment process, including time, were also taken from the clinical records. Trained research nurses in each institution carried out the entire process of screening, selection, and collection of information by means of a standardized form. The clinical researchers performed a permanent review and verification of the patients included and the data collected. Patients were followed until hospital discharge.

\section{Statistical analysis \\ Propensity matching}

To generate the propensity score (PS) [8], two logistic regression models were made for the use of antimicrobials in the first hour and $3 \mathrm{~h}$ after admission to the ED, respectively. To test the fit of the models, we used the adjusted pseudo- $R^{2}$ ( 1 = perfect fit of the data to the model), the Hosmer-Lemeshow goodness-of-fit statistic $(p>0.05)$ and the area under the ROC curve (AUC - ROC Curve) (0.5 = non-discrimination, $1=$ perfect discrimination $)$. Under these criteria, the model, which included 28 variables related to the clinical care of the patients as well as direct measurements of physiological derangement, was selected (Additional files 1 and 2), as all of them may prognostic factors associated with both the outcome and the likelihood of being exposed to the intervention [16]. With the PS derived from these variables, the nearest neighbor 1:1 match was used, selecting for each treated individual the control individual with the shortest distance according to local optimal algorithms (greedy algorithms), no caliper and no replacement [17]. A standardized mean difference (SMD) < 10\% was considered an adequate balance in the distribution between the groups.

For the evaluation of the pre- and post-matching groups in the continuous variables, median and interquartile ranges were measured, which were compared by means of the Mann-Whitney $U$ test; and categorical variables were described with proportions, compared by Pearson's chi-square test.

\section{Outcome models}

Finally, as a sensitivity analysis, several logistic regression models were performed both in the population matched by propensity score and in the total population. These models were on the general population and in subgroups by (1) confirmed diagnosis of infection, (2) shock (both by previous definitions [12]: SBP $<90 \mathrm{mmHg}$ or MAP $<65 \mathrm{mmHg}$ after fluid challenge or LA > $4 \mathrm{mmol} / \mathrm{l}$; and by Sepsis-3 [18]: SBP $<90$ $\mathrm{mmHg}$ or $\mathrm{MAP}<65 \mathrm{mmHg}$ and use of vasopressors and LA > $2 \mathrm{mmol} / \mathrm{l}$ ), and (3) appropriate use of antimicrobials. In all these models, the crude effect of time of antimicrobial administration was estimated, as well as the effect adjusting for the propensity score and for confounding covariates such as age, Charlson Index, intravenous fluids, blood cultures in the first 3 $h$, lactate value, the SOFA, and the APACHE II scores. In addition, we analyzed the effect of each hour of delay in the administration of antimicrobials as a continuous independent variable for all previous regression models among the total study population. There was $2.2 \%$ of missing data, which were excluded for the generation of the propensity score. Statistical analyses were performed with the STATA version 14 
Program (StataCorp, College Station, TX) and R version 3.4.1. The full paper and the data followed the STROBE statement [19].

\section{Results}

Baseline characteristics before propensity matching

A total of 5022 patients were screened, of which 2454 entered the study (Fig. 1). The clinical, physiological, and laboratory parameters of the pre- and post-matched population are presented according to the administration of antimicrobials within the first hour (Table 1) or $3 \mathrm{~h}$ (Table 2). The additional files 1 and 2 include all the variables taken into account for the PS model.

The median age of the patients was 62 years $(\mathrm{IQR}=$ $46-74)$ and $50 \%(n=1227)$ were women. The most frequent diagnoses at admission were urinary tract infection (UTI) and pneumonia with $27.8 \%(n=682)$ and $27.3 \%(n=671)$, respectively. Altogether, $2140(87 \%)$ of the patients had blood cultures, of which $29.4 \%(n=$ 629) were positive; the most frequent microorganisms were Escherichia coli (36.6\%; $n=230)$, Staphylococcus aureus (21\%; $n=132)$, and Klebsiella pneumoniae $(10.2 \% ; n=64)$. A $76.5 \%(n=1637)$ of the blood cultures were taken prior to the start of the antimicrobial, $90.3 \%$ $(n=2215)$ of the patients received antimicrobials in the first $24 \mathrm{~h}$ and started at a median of $5 \mathrm{~h}$ from the time of arrival at the ED (IQR $=2-9), 31.8 \%(n=781)$ in the first $3 \mathrm{~h}$ and $13.9 \%(n=340)$ in the first hour of admission to the ED. The most commonly used antimicrobials were piperacillin/tazobactam in $53.7 \%(n=1189)$, ampicillin/sulbactam in $10.1 \%(n=223)$, and meropenem in $6.6 \%(n=145)$. The diagnosis of infection was confirmed in 1909 patients $(77.8 \%)$ and among them, $23.5 \%(n=$
449) had an inadequate prescription, according to microbiological or clinical criteria. Some clinical care variables in the first hour of administration of antimicrobials were central venous catheter placement $(10.9 \%$ vs. $6.8 \%, p=0.008)$, red blood cell transfusion ( $4.1 \%$ vs. $1.8 \%, p=0.007)$, onset of vasopressors in the first $24 \mathrm{~h}$ (27.4\% vs. $14.1 \%, p=<0.001$ ), and request of blood cultures $(94.4 \%$ vs. $86.1 \%, p=<0.001)$. Among the total cohort, $42.3 \%$ of the patients $(n=1038)$ were admitted to the intensive care unit (ICU) and $15.3 \%(n=376)$ required mechanical ventilation. When applying definitions for the identification of patients with septic shock, it was found that $35.4 \%(n=869)$ and $7.3 \%(n=179)$ met the older and newest diagnostic criteria, respectively. The total cohort in-hospital mortality rate was $11.5 \%(n=283)$; divided by antimicrobial administration time, it was $<1 \mathrm{~h} 12.7 \%(n=43),<3 \mathrm{~h} 13.3 \%(n=104)$, and $>3 \mathrm{~h} 10.7 \%(n=179)$.

The SMD was much larger than $10 \%$ in most of the variables analyzed in the unmatched population, both within the first hour or within $3 \mathrm{~h}$ (Tables 1 and 2). The variables SBP, amount of fluids in the first $6 \mathrm{~h}$, urinary output, use of vasopressors, and admission to ICU, seems to indicate that the population that received the antimicrobial earlier was more critically ill.

\section{Baseline characteristics after propensity matching}

After matching for the propensity score, a population of 340 patients with antimicrobials within the first hour and 340 with subsequent antimicrobial treatment was obtained (Table 1). The same pairing procedure by propensity score obtained a population of 781 patients with

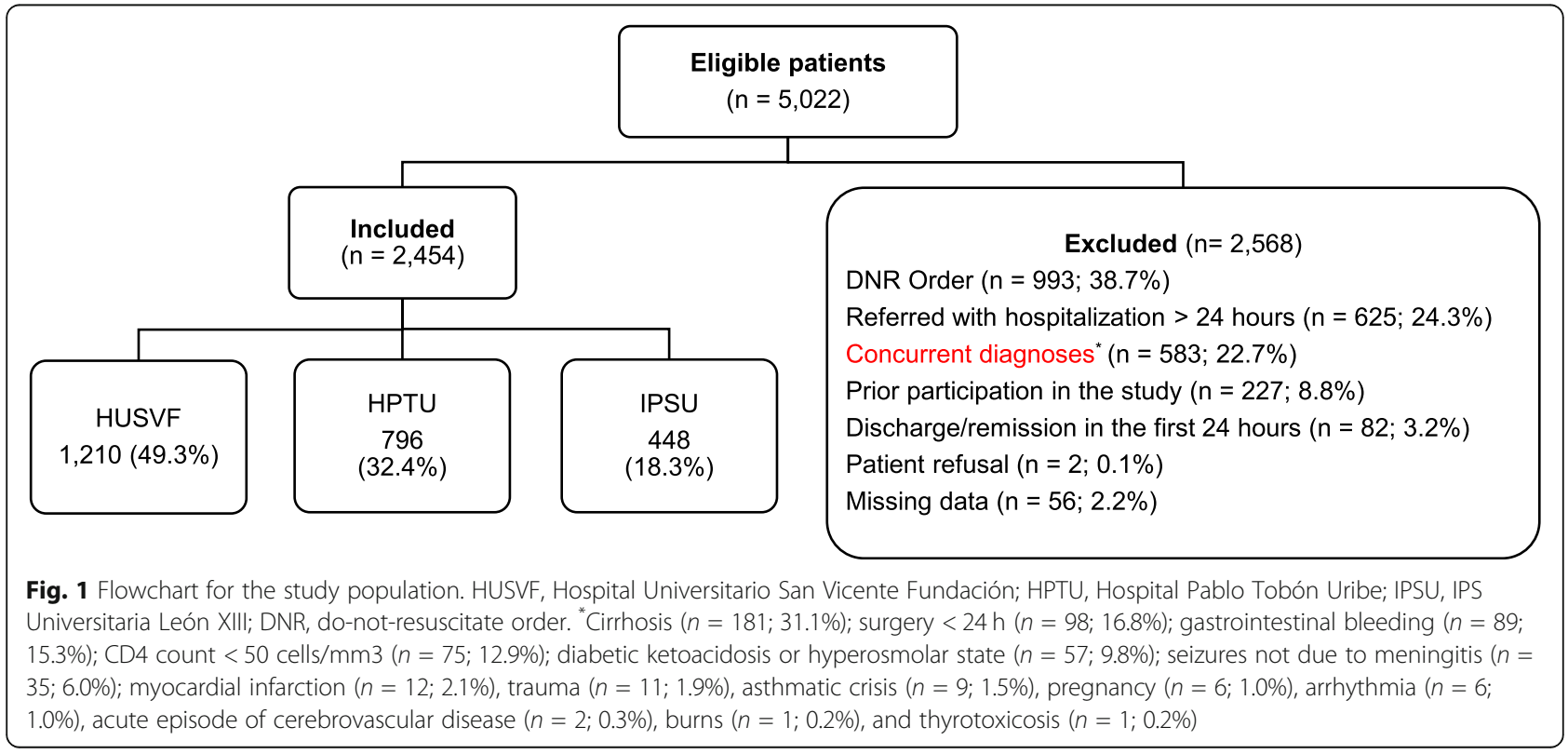


Table 1 Use of antimicrobials within the first hour of admission to the ED, with and without propensity score matching

\begin{tabular}{|c|c|c|c|c|c|c|c|c|}
\hline \multirow[t]{2}{*}{ Variable } & \multicolumn{4}{|l|}{ Pre-match } & \multicolumn{4}{|l|}{ Post-match } \\
\hline & $\begin{array}{l}>1 \mathrm{~h}, \boldsymbol{n}=2114 \\
(86.2 \%)\end{array}$ & $\begin{array}{l}<1 \mathrm{~h}, \boldsymbol{n}=340 \\
(13.8 \%)\end{array}$ & $\boldsymbol{p}$ value & SMD (\%) & $\begin{array}{l}>1 \mathrm{~h}, \boldsymbol{n}=340 \\
(50 \%)\end{array}$ & $\begin{array}{l}<1 \mathrm{~h}, \boldsymbol{n}=340 \\
(50 \%)\end{array}$ & $\boldsymbol{p}$ value & SMD (\%) \\
\hline Age & $62(48-75)$ & $59(42-70)$ & $<0.001$ & 27.4 & $60(43-71)$ & $59(42-70)$ & 0.757 & 1.3 \\
\hline Systolic blood pressure & $110(90-131)$ & $89(80-115)$ & $<0.001$ & 72.5 & $95(82-116)$ & $89(80-115)$ & 0.037 & 9.9 \\
\hline Temperature ${ }^{\circ} \mathrm{C}$ & $37(36.7-38.3)$ & $37.8(37-38.7)$ & $<0.001$ & 34.7 & $37.5(37-38.7)$ & $37.8(37-38.7)$ & 0.462 & 6.9 \\
\hline Heart rate & $103(88-117)$ & $110(99-123)$ & $<0.001$ & 43.7 & $110(96-120)$ & $110(99-123)$ & 0.274 & 9.9 \\
\hline Respiratory rate & $19(18-22)$ & $21(18-25)$ & $<0.001$ & 38 & $20(18-23)$ & $21(18-25)$ & 0.027 & 12.9 \\
\hline Lactate & $2.5(1.5-3.5)$ & $2.3(1.3-3.4)$ & 0.086 & 8.2 & $2.1(1.2-3.2)$ & $2.3(1.3-3.4)$ & 0.139 & 8.8 \\
\hline Central venous catheter & $144(6.8 \%)$ & 37 (10.9\%) & 0.008 & 9.9 & $29(8.5 \%)$ & 37 (10.9\%) & 0.300 & 4.3 \\
\hline Fluids (IVF) in the first $6 \mathrm{~h}$ & 1608 (76.1\%) & $313(92.1 \%)$ & $<0.001$ & 40.5 & $314(92.4 \%)$ & $313(92.1 \%)$ & 0.886 & 0.5 \\
\hline Amount of fluids in $6 \mathrm{~h}$ & $955(150-1500)$ & $1500(637-2325)$ & $<0.001$ & 63.3 & $1450(500-2150)$ & $1500(637-2325)$ & 0.290 & 15.5 \\
\hline Urinary output & $646(30.6 \%)$ & $171(50.3 \%)$ & $<0.001$ & 39.9 & $159(46.8 \%)$ & $171(50.3 \%)$ & 0.357 & 5.8 \\
\hline Vasopressors & $299(14.1 \%)$ & $93(27.4 \%)$ & $<0.001$ & 28.2 & $84(24.7 \%)$ & $93(27.4 \%)$ & 0.432 & 4.5 \\
\hline Blood cultures & 1819 (86.1\%) & $321(94.4 \%)$ & $<0.001$ & 21.9 & $324(95.3 \%)$ & $321(94.4 \%)$ & 0.603 & 1.7 \\
\hline $\begin{array}{l}\text { Blood cultures taken prior to } \\
\text { the beginning of antibiotics }\end{array}$ & $1508(71.3 \%)$ & $129(37.9 \%)$ & $<0.001$ & 68.5 & $138(40.6 \%)$ & 129 (37.9\%) & 0.480 & 4.4 \\
\hline Admission to ICU/SCU & $856(40.5 \%)$ & $182(53.5 \%)$ & $<0.001$ & 26.4 & $179(52.7 \%)$ & $182(53.5 \%)$ & 0.818 & 1.4 \\
\hline Mechanical ventilation & $311(14.7 \%)$ & $65(19.1 \%)$ & 0.036 & 9.9 & $63(18.5 \%)$ & $65(19.1 \%)$ & 0.844 & 1 \\
\hline $\begin{array}{l}\text { Confirmed diagnosis of } \\
\text { infection }\end{array}$ & 1624 (76.8\%) & $285(83.8 \%)$ & 0.004 & 16.2 & $263(77.4 \%)$ & $285(83.8 \%)$ & 0.033 & 11.5 \\
\hline Inadequate antimicrobials & $610(28.9 \%)$ & $42(12.4 \%)$ & $<0.001$ & 39.6 & $45(13.2 \%)$ & $42(12.4 \%)$ & 0.731 & 1.6 \\
\hline Mortality* & $240(11.4 \%)$ & $43(12.7 \%)$ & 0.488 & 3.1 & $40(11.8 \%)$ & $43(12.7 \%)$ & 0.725 & 1.6 \\
\hline Hospital stay & $10(6-16)$ & $11(6-19)$ & 0.002 & 21.2 & $11(6-19)$ & $11(6-19)$ & 0.335 & 2.3 \\
\hline SOFA score* & $4(2-6)$ & $5(3-7)$ & $<0.001$ & 43.2 & $4(3-7)$ & $5(3-7)$ & 0.473 & 9.2 \\
\hline APACHE II score* & $14(9-12)$ & $15(11-19)$ & $<0.001$ & 32.4 & 15 (9-18) & $15(11-19)$ & 0.148 & 14.0 \\
\hline Charlson Index* & $1(0-2)$ & $1(0-2)$ & 0.010 & 18.2 & $1(0-2)$ & $1(0-2)$ & 0.430 & 2.2 \\
\hline $\begin{array}{l}\text { h between admission and } \\
\text { administration of antibiotics* }\end{array}$ & $6(4-10)$ & $1(1-1)$ & $<0.001$ & - & $4(2-8)$ & $1(1-1)$ & $<0.001$ & - \\
\hline
\end{tabular}

The measurements for continuous variables are the median (IQR) and for categorical: $n$ (\%). SMD standardized mean difference

*The variables were not included to generate the propensity score

antimicrobials within the first $3 \mathrm{~h}$ and 781 with subsequent antimicrobial treatment (Table 2).

For the first hour comparison, SMD was $<10 \%$ for most variables after PS matching, except respiratory rate (12.9\%), creatinine (11.9\%), amount of fluids in the first $6 \mathrm{~h}(15.5 \%)$, and confirmed diagnosis of infection $(11.5 \%)$. On the other hand, for the first $3 \mathrm{~h}$, the variables with SMD > 10\% after matching by PS were systolic blood pressure (20.1\%), amount of fluids in the first $6 \mathrm{~h}(21.3 \%)$, urinary output $(12.5 \%)$, blood cultures taken prior to the beginning of antibiotics $(23.8 \%)$, and site of infection (10.7\%).

\section{Outcome models}

\section{General model}

For the matched population, in the general model adjusting for propensity score and confounding covariates, there were no significant differences in mortality with antimicrobial administration within or after the first hour ( $\leq 1 \mathrm{~h}$ vs $>1 \mathrm{~h})$ (OR 1.03 ; 95\% CI = 0.63; $1.70)$ nor within the first $3 \mathrm{~h}$ or after ( $\leq 3 \mathrm{~h}$ vs $>3 \mathrm{~h}$ ) (OR $0.85 ; 95 \% \mathrm{CI}=0.61 ; 1.20)$ (Table 3$)$.

\section{Subgroups models}

Similar results were found when analyzing the population with confirmed infection, adequate use of antibiotics, and septic shock according to two different definitions (Table 3). In addition, it was not possible to demonstrate a significant increase in-hospital mortality for each hour of delay in the administration of antimicrobials, nor in the crude analysis (OR $=0.98 ; 95 \% \mathrm{CI}=0.96 ; 1.01)$, nor in the analysis adjusted for covariates $(\mathrm{OR}=1.00 ; 95 \% \mathrm{CI}=0.97$; 1.03) (Table 4). 
Table 2 Use of antimicrobials within the $3 \mathrm{~h}$ of admission to the ED, with and without propensity score matching

\begin{tabular}{|c|c|c|c|c|c|c|c|c|}
\hline \multirow[t]{2}{*}{ Variable } & \multicolumn{4}{|l|}{ Pre-matching } & \multicolumn{4}{|l|}{ Post-matching } \\
\hline & $\begin{array}{l}>3 \mathrm{~h}, \boldsymbol{n}=1673 \\
(68.2 \%)\end{array}$ & $\begin{array}{l}<3 \mathrm{~h}, \boldsymbol{n}=781 \\
(31.8 \%)\end{array}$ & $\boldsymbol{p}$ value & SMD (\%) & $\begin{array}{l}>3 h, \boldsymbol{n}=781 \\
(50 \%)\end{array}$ & $\begin{array}{l}<3 h, \boldsymbol{n}=781 \\
(50 \%)\end{array}$ & $\boldsymbol{p}$ value & SMD (\%) \\
\hline Age & $63(47-75)$ & $60(45-72)$ & 0.004 & 15.9 & $61(45-73)$ & $60(45-72)$ & 0.541 & 3 \\
\hline Systolic blood pressure & $115(93-134)$ & $93(80-119)$ & $<0.001$ & 73.6 & $100(85-124)$ & $93(80-119)$ & $<0.001$ & 20.1 \\
\hline Temperature ${ }^{\circ} \mathrm{C}$ & $37(36.7-38.3)$ & $37.3(37-38.5)$ & $<0.001$ & 17.6 & $37.2(36.9-38.5)$ & $37.3(37-38.5)$ & 0.318 & 4.6 \\
\hline Heart rate & $102(88-116)$ & $110(95-120)$ & $<0.001$ & 38.2 & $106(90-120)$ & $110(95-120)$ & 0.123 & 10.1 \\
\hline Respiratory rate & $19(18-22)$ & $20(18-25)$ & $<0.001$ & 39.3 & $20(18-24)$ & $20(18-25)$ & 0.002 & 10.6 \\
\hline Lactate & $2.6(1.6-3.5)$ & $2.3(1.3-3.4)$ & $<0.001$ & 1.9 & $2.4(1.3-3.6)$ & $2.3(1.3-3.4)$ & 0.354 & 0.5 \\
\hline Central venous catheter & $88(5.3 \%)$ & $93(11.9 \%)$ & $<0.001$ & 12.8 & $71(9.1 \%)$ & $93(11.9 \%)$ & 0.069 & 5.1 \\
\hline Fluids (IVF) in the first $6 \mathrm{~h}$ & $1212(72.4 \%)$ & 709 (90.8\%) & $<0.001$ & 36 & 704 (90.1\%) & 709 (90.8\%) & 0.667 & 1.2 \\
\hline Amount of fluids in $6 \mathrm{~h}$ & $660(0-1500)$ & $1420(550-2300)$ & $<0.001$ & 70.6 & $1210(500-2000)$ & $1420(550-2300)$ & 0.006 & 21.3 \\
\hline Urinary output & $451(27 \%)$ & $366(46.9 \%)$ & $<0.001$ & 34.1 & 306 (39.2\%) & $366(46.9 \%)$ & 0.002 & 12.5 \\
\hline Vasopressors & $190(11.4 \%)$ & $202(26.9 \%)$ & $<0.001$ & 25.9 & $156(20 \%)$ & $202(25.9 \%)$ & 0.006 & 10 \\
\hline Blood cultures & $1411(84.3 \%)$ & $729(93.3 \%)$ & $<0.001$ & 18.1 & $714(91.4 \%)$ & 729 (93.3\%) & 0.153 & 3.6 \\
\hline $\begin{array}{l}\text { Blood cultures taken prior to } \\
\text { the beginning of antibiotics }\end{array}$ & $1216(72.7 \%)$ & $421(53.9 \%)$ & $<0.001$ & 32.1 & $535(68.5 \%)$ & $421(53.9 \%)$ & $<0.001$ & 23.8 \\
\hline Admission to ICU/SCU & $628(37.5 \%)$ & $410(52.5 \%)$ & $<0.001$ & 25.6 & $377(48.3 \%)$ & $410(52.5 \%)$ & 0.095 & 6.9 \\
\hline Mechanical ventilation & $217(13 \%)$ & $159(20.4 \%)$ & $<0.001$ & 13.5 & 149 (19.1\%) & 159 (20.4\%) & 0.525 & 2.2 \\
\hline $\begin{array}{l}\text { Confirmed diagnosis of } \\
\text { infection }\end{array}$ & $1266(75.7 \%)$ & $643(82.3 \%)$ & $<0.001$ & 12.4 & 617 (79\%) & $643(82.3 \%)$ & 0.096 & 5.9 \\
\hline Inadequate antimicrobials & $527(31.5 \%)$ & $125(16 \%)$ & $<0.001$ & 29.1 & 149 (19.1\%) & $125(16 \%)$ & 0.110 & 5.5 \\
\hline Mortality* & 179 (10.7\%) & $104(13.3 \%)$ & 0.059 & 5 & 105 (13.4\%) & 104 (13.3\%) & 0.941 & 0.2 \\
\hline Hospital stay & $10(6-16)$ & $11(6-18)$ & $<0.001$ & 21.2 & $10(6-17)$ & $11(6-18)$ & 0.153 & 7.5 \\
\hline SOFA score* & $3(2-5)$ & $4(3-7)$ & $<0.001$ & 48.5 & $4(3-6)$ & $4(3-7)$ & 0.034 & 17.7 \\
\hline APACHE $\|$ score* & $13(9-18)$ & $15(10-19)$ & $<0.001$ & 31.5 & $14(10-19)$ & $15(10-19)$ & 0.052 & 13.2 \\
\hline Charlson Index* & $1(0-2)$ & $1(0-2)$ & 0.010 & 16.2 & $1(0-2)$ & $1(0-2)$ & 0.101 & 6.4 \\
\hline
\end{tabular}

The measurements for continuous variables are the median (IQR) and for categorical: $n(\%)$. SMD standardized mean difference *The variables were not included to generate the propensity score

\section{Total population}

When analyzing the total unmatched population, none of these different models showed significant differences in mortality with the administration of antimicrobials within $1 \mathrm{~h}$ or $3 \mathrm{~h}$ (Additional file 3 ).

\section{Discussion}

In this secondary analysis of a multicenter prospective cohort of patients admitted to the emergency department with suspected sepsis, septic shock, or infection with organ dysfunction, the early administration of antimicrobials was not associated with a significant decrease in in-hospital mortality when comparing different time cut offs ( $\leq 1 \mathrm{~h}$ vs $>1 \mathrm{~h}$ nor $\leq 3 \mathrm{~h}$ vs $>3 \mathrm{~h}$ ). Nor was an association found between each hour of delay in the administration of antimicrobials and an increase in mortality.

These results appear to be contrary to current recommendations, in which the administration of appropriate antibiotics within the first hour of recognition of sepsis is considered a standard of management quality [20]. The foregoing has been based on several observational studies that indicate that the delay in antibiotic administration is associated with higher mortality [21-24]. A retrospective study of a large administrative database that included 35,000 patients with sepsis, severe sepsis, and septic shock concluded that every hour of delay in the administration of antibiotics is associated with a higher probability of hospital mortality adjusted for the characteristics of the patient and the severity of the disease (OR, 1.09; 95\% CI, 1.05-1.13); although, it is important to consider that in this study, no data were presented on confirmation of infection nor adequacy of antimicrobials [21]. In the study by Seymour et al., of 49, 331 patients with severe sepsis or septic shock, an association was also found between the time elapsed in the administration of antibiotics and hospital mortality (OR, 1.04 per hour; 95\% CI, 1.03-1.06) [22]. Reinforcing these findings, in a retrospective cohort with 5072 patients, Pruinelli et al. found that a 125-min delay in the onset 
Table 3 Effect of the antimicrobial administration either within 1 or 3 hours of admission to the ED, matched by PS, on in-hospital mortality

\begin{tabular}{|c|c|c|}
\hline Models & $\begin{array}{l}\text { Antimicrobials } \leq 1 \mathrm{~h} \text { vs. }>1 \mathrm{~h}, \mathrm{OR}(95 \% \mathrm{Cl}) \\
n=680\end{array}$ & $\begin{array}{l}\text { Antimicrobials } \leq 3 \mathrm{~h} \text { vs. }>3 \mathrm{~h}, \mathrm{OR}(95 \% \mathrm{Cl}) \\
n=1562\end{array}$ \\
\hline No adjustment & $1.09(0.69 ; 1.72)$ & $0.98(0.74 ; 1.32)$ \\
\hline Adjusted for propensity score & $1.05(0.66 ; 1.67)$ & $0.86(0.63 ; 1.17)$ \\
\hline Adjusted for covariates* & $1.07(0.65 ; 1.75)$ & $0.93(0.67 ; 1.28)$ \\
\hline Adjusted for propensity score + covariates * & $1.03(0.63 ; 1.70)$ & $0.85(0.61 ; 1.20)$ \\
\hline Patients with confirmed infection & $n=548$ & $n=1260$ \\
\hline No adjustment & $1.00(0.61 ; 1.64)$ & $1.00(0.73 ; 1.38)$ \\
\hline Adjusted for propensity score & $0.99(0.61 ; 1.63)$ & $0.89(0.64 ; 1.25)$ \\
\hline Adjusted for covariates** & $0.92(0.54 ; 1.58)$ & $0.91(0.64 ; 1.30)$ \\
\hline Adjusted for propensity score + covariates** & $0.90(0.53 ; 1.56)$ & $0.88(0.61 ; 1.26)$ \\
\hline Patients with adequate antibiotics & $n=593$ & $n=1288$ \\
\hline No adjustment & $0.96(0.59 ; 1.56)$ & $0.98(0.71 ; 1.36)$ \\
\hline Adjusted for propensity score & $0.93(0.57 ; 1.53)$ & $0.82(0.58 ; 1.16)$ \\
\hline Adjusted for covariates*** & $0.88(0.51 ; 1.50)$ & $0.89(0.62 ; 1.27)$ \\
\hline Adjusted for propensity score + covariates*** & $0.86(0.50 ; 1.46)$ & $0.82(0.56 ; 1.19)$ \\
\hline Patients with shock—Rivers & $n=342$ & $n=702$ \\
\hline No adjustment & $0.96(0.54 ; 1.73)$ & $0.98(0.66 ; 1.44)$ \\
\hline Adjusted for propensity score & $0.91(0.50 ; 1.66)$ & $0.87(0.57 ; 1.32)$ \\
\hline Adjusted for covariates* & $0.92(0.49 ; 1.74)$ & $0.92(0.59 ; 1.42)$ \\
\hline Adjusted for propensity score + covariates* & $0.87(0.46 ; 1.66)$ & $0.85(0.54 ; 1.35)$ \\
\hline Patients with shock-Sepsis-3 & $n=91$ & $n=170$ \\
\hline No adjustment & $0.85(0.34 ; 2.15)$ & $0.74(0.39 ; 1.43)$ \\
\hline Adjusted for propensity score & $0.70(0.26 ; 1.84)$ & $0.56(0.27 ; 1.15)$ \\
\hline Adjusted for covariates* & $0.86(0.31 ; 2.37)$ & $0.86(0.40 ; 1.85)$ \\
\hline Adjusted for propensity score + covariates* & $0.75(0.26 ; 2.14)$ & $0.65(0.28 ; 1.51)$ \\
\hline
\end{tabular}

${ }^{*}$ Covariates: age, Charlson Index, intravenous fluids $\geq 1500$ first hour, blood cultures in the first $3 \mathrm{~h}$, lactate, SOFA score, APACHE II score, confirmed diagnosis of infection, and inadequate antimicrobials

${ }^{* *}$ Covariates: age, Charlson Index, intravenous fluids $\geq 1500$ first hour, blood cultures in the first $3 \mathrm{~h}$, lactate, SOFA score, APACHE II score, and inadequate antimicrobials

${ }^{* * *}$ Covariates: age, Charlson Index, intravenous fluids $\geq 1500$ first hour, blood cultures in the first $3 \mathrm{~h}$, lactate, SOFA score, APACHE II score, and confirmed diagnosis of infection

of antibiotics increases the risk of mortality, concluding that very short delays could have an adverse impact on outcomes [25]. A recent systematic review concluded that the benefit of antibiotic use within the first hour was greater in patients with septic shock [26]. Additionally, supporting the benefit of early antimicrobials in those most critically ill patients, in our primary analysis that included 884 adult patients admitted to the ED with infection and shock according to the previous definition (systolic blood pressure $<90 \mathrm{mmHg}$ after fluids challenge or lactate $>4 \mathrm{mmol} / \mathrm{L}$ ), we found a potential absolute reduction in mortality of $21 \%$ with the use of antibiotics in the first $3 \mathrm{~h} \mathrm{[10].} \mathrm{However,} \mathrm{in} \mathrm{that} \mathrm{analysis,}$ we used an instrumental variable approach for comparison among all of the components of the full EGDT protocol, trying to establish the weight of each one in the outcomes of patients.

Although the usefulness of antimicrobials is not in doubt, the controversy persists as to whether the evidence is strong enough to support current early administration guidelines. In this regard, it is stated that the evidence for "every hour of delay" is based solely on retrospective analyses of administrative databases and it is emphasized that these designs are missing crucial data, such as the confirmation of infection, the appropriate choice of antimicrobials with their doses, and control of the source of infection, important confounding factors with possible influence on outcomes [27]. In a prospective and multicenter study by de Groot et al, 1168 patients were stratified in three categories of severity 
Table 4 Effect of each hour of delay in the administration of antimicrobials since admission to the ED on in-hospital mortality

\begin{tabular}{lll}
\hline Models & \multicolumn{1}{l}{ Type of logistic regression } & Adjusted for covariates \\
\cline { 2 - 3 } & No adjustment & OR $(95 \% \mathrm{Cl})$ \\
\hline Models with total population $(n=2215)$ & OR $(95 \% \mathrm{Cl})$ & $1.00^{*}(0.97 ; 1.03)$ \\
Models with confirmed infection $(n=1751)$ & $0.98(0.96 ; 1.01)$ & $1.00^{* *}(0.97 ; 1.03)$ \\
Models with not confirmed infection $(n=464)$ & $0.98(0.96 ; 1.01)$ & $1.03^{* *}(0.95 ; 1.10)$ \\
Models with adequate antibiotics $(n=1802)$ & $1.00(0.94 ; 1.07)$ & $1.01^{* * *}(0.97 ; 1.03)$ \\
Models with not adequate antibiotics $(n=413)$ & $0.98(0.95 ; 1.01)$ & $1.01^{* * *}(0.95 ; 1.07)$ \\
Models with shock-Rivers $(n=830)$ & $0.99(0.94 ; 1.05)$ & $1.01^{*}(0.96 ; 1.05)$ \\
Models with shock-Sepsis-3 $(n=179)$ & $0.99(0.96 ; 1.03)$ & $1.03^{*}(0.94 ; 1.12)$ \\
\hline
\end{tabular}

"Covariates: age, Charlson Index, intravenous fluids $\geq 1500$ first hour, blood cultures in the first $3 \mathrm{~h}$, lactate, SOFA score, APACHE II score, confirmed diagnosis of infection, and inadequate antimicrobials

${ }^{* *}$ Covariates: age, Charlson Index, intravenous fluids $\geq 1500$ first hour, blood cultures in the first $3 \mathrm{~h}$, lactate, SOFA score, APACHE II score, and inadequate antimicrobials

${ }^{* * *}$ Covariates: age, Charlson Index, intravenous fluids $\geq 1500$ first hour, blood cultures in the first $3 \mathrm{~h}$, lactate, SOFA score, APACHE II score, and confirmed diagnosis of infection

according to the predisposition, infection, response, and organ failure score (PIRO, score 1 to 7,8 to 14 and $>14$ points), without finding a reduction in mortality according to the time of onset of the antibiotics in any of the categories [28]. In a before-and-after study conducted in patients with suspected infection in a surgical intensive care unit (ICU), Hranjec et al. compared an aggressive regimen of antibiotic administration (initiation of antibiotics in all patients with suspected infection) with a conservative regimen (starting only after objective confirmation of infection), finding that the former was associated with higher mortality (OR, 2.5; 95\% CI, 1.5-4.0) [7]. In addition, in a prospective observational study that enrolled 1184 adult patients diagnosed with severe sepsis and septic shock, Abe et al. were unable to show a linear relationship between the timing of antibiotic administration, such as within $1 \mathrm{~h}$ or $3 \mathrm{~h}$ after sepsis recognition, and in-hospital mortality, nor a relation between time to antibiotics as a continuous variable and mortality (OR, 0.99; 95\% CI 0.991.0; $p=0.152$ ) [29]. In our knowledge, the only randomized controlled trial (RCT) that evaluated early antimicrobials administration in 2672 patients with suspected infection, although in a prehospital setting, failed to find an association between early administration of antimicrobials and mortality $(\mathrm{RR}=0.95$; 95\% CI, 0.74-1.24) [30].

Additionally, our results also agree with the conclusions of the systematic review by Sterling et al., who evaluated two scenarios. In the first scenario, the authors compared patients who received antibiotics 3 or more hours after admission to the emergency room with those who received antibiotics within the first $3 \mathrm{~h}$, without finding a significant increase in mortality (OR, 1.16; $95 \%$ CI, 0.92-1.46). For the second scenario, they compared the administration after $1 \mathrm{~h}$ of recognizing of the shock with the administration in the first hour, also indicating that early administration is not associated with a significant benefit in mortality (OR, 1.46; 95\% CI, 0.892.40) [6]. In this regard, it should be noted that in our study, when doing a subgroup analysis, we did not find significant differences in mortality with the administration of antimicrobials within $1 \mathrm{~h}$ or within $3 \mathrm{~h}$ of admission to the ED in patients with septic shock, in contrast to emerging literature that suggests a greater benefit in this population $[21,22,26]$. These findings are similar to the results of a recent small observational study that included 150 septic shock patients admitted to the medical intensive care unit (MICU) with an in-hospital mortality rate of 49.3\%; this study did not show an association between timing of antibiotic administration and mortality [31]. All this would suggest a more prudent-albeit critical-approach to the consideration of the timely and adequate initiation of antimicrobials, in which the initial resuscitation measures and the determination of the focus of infection would be critical.

Among the strengths of our study is its character as a multicenter prospective cohort, and not based on an administrative database, with a fairly representative population of the heterogeneous clinical presentation of sepsis in the ED, in addition to a reliable collection of crucial data about the confirmation of infection, the administration time of the antimicrobials, and the adequacy of their prescription, based on the resistance profile of the microorganisms. In the analysis, in addition to the exhaustive adjustment for various covariates, novel statistical techniques like the PS were considered to try to achieve a balance of the groups and estimate more accurately the effect of the intervention, given the logistical and ethical difficulty to perform a RCT in the field.

\section{Limitations}

It is necessary to highlight the restriction regarding the sample size, for which it is not possible to rule out a 
beneficial effect, although it is important to note that our sample size is one of the largest collected prospectively in a study that shows no association between early administration of antimicrobials and mortality in patients with sepsis [7, 28-32]. Also, the problem of residual confounding-despite the use of the propensity score and adjustment for covariates-cannot be eliminated. With regard to the definitions of antimicrobial administration time (time zero), our reference to the delay in administration was from admission to the ED, which we consider more relevant from the clinical point of view, while in some previous research it is conceived from the onset of hypotension or recognition of shock [33]. In addition, in our study, we only evaluate in-hospital mortality, so we cannot conclude anything about the longterm effects, a topic analyzed in other research [34, 35]. Another consideration is that, during the recruitment period of the study population, the Sepsis-3 criteria were not available. However, in the current study, we analyzed the eligible population with any organ dysfunction, similar to the current process based on the SOFA score. Indeed, the median SOFA score in our cohort was 4 or 5 for antibiotics $>$ or $<1 \mathrm{~h}$, respectively.

The overall mortality rate in our study was lower (11.5\%), compared to previous, retrospective (56\%) [33] and prospective (19\%) [32] studies, which may decrease the power to detect differences; the relatively high rates of UTI (27.8\%) compared to other studies might account for this lower rate. Nevertheless, it is important to highlight that previous studies had a lower mortality rate in patients with diagnosis of sepsis (3.3\%) and severe sepsis (8.8\%) [21]. In addition, in our study, only $\sim 50 \%$ of patients who received antimicrobials within the first hour were admitted to the ICU and about $25 \%$ received vasopressors. Thus, it appears that the present cohort had a lower severity of illness than previous studies, as well as a lower proportion of patients with septic shock, population in which the literature seems to suggest a greater benefit from the early administration of antimicrobials $[10,21,22,26]$.

\section{Interpretation and implications}

The benefit of early administration in this last population perhaps was not reflected in our study due to the aforementioned limitations, so a cautious interpretation of these findings is necessary, since we do not suggest delaying the use of antimicrobials in patients with septic shock despite the current moderate evidence. However, another is the scenario for patients with sepsis and suspected sepsis, a population that is represented in our study in the daily context of an ED and which has also been matched by multiple covariates and confounding factors. In this population without shock, the association between the early administration of antimicrobials and mortality has been null or minimal, a situation also evidenced in our study [21, 22, 30]. If we take this into account, the most recent recommendations, in which the rapid and aggressive use of antimicrobials is done even in patients with no infection to simply meet a benchmark, would not be justified in this context, where some clinical important information could be collected to ensure the presence of infection without increased risk of adverse outcomes [36].

\section{Conclusion}

In the present study, it was not possible to demonstrate a statistically significant association between the early administration of antimicrobials and mortality in sepsis patients with or without shock. However, according to the wide confidence interval, we cannot discard a beneficial effect of earlier antibiotics in patients with septic shock. These findings reinforce the idea that more research is needed in the field and suggest a more comprehensive approach to sepsis and its treatment, considering early detection, multiple interventions, and goals beyond the simple time of antimicrobial administration, which may favor the indiscriminate use of them.

\section{Supplementary information}

Supplementary information accompanies this paper at https://doi.org/10. 1186/s40560-020-00448-1.

Additional file 1. Use of antimicrobials within the first hour of admission to the ED, with and without propensity score matching.

Additional file 2. Use of antimicrobials within the three hours of admission to the ED, with and without propensity score matching.

Additional file 3. Effect of the antimicrobial administration in the first hours of admission to the ED on in-hospital mortality. Models with total population.

\section{Abbreviations}

ED: Emergency department; IQR: Interquartile range; OR: Odds ratio; HUSVF: Hospital Universitario San Vicente Fundación; HPTU: Hospital Pablo Tobón Uribe; IPSU: IPS Universitaria León XIII; CDC: Center for Disease Control and Prevention; CR: Creatinine; INR: International normalized ratio; PTT: Partial thromboplastin time; PLT: Platelet count; LA: Lactic acid; SBP: Systolic blood pressure; MAP: Mean arterial pressure; PS: Propensity score; SMD: Standardized mean difference; ICU: Intensive care unit

\section{Acknowledgements}

None

\section{Authors' contributions}

All authors contributed to the conception of the research question and design of the study. JA and FJ contributed to the analysis and interpretation. All authors wrote the article and all the authors substantially contributed to its revision and approval.

\section{Funding}

Funded by the Colombian Agency of Science, Technology and Innovation (COLCIENCIAS) (code 111556933362) and the University of Antioquia (Code 2582).

Availability of data and materials

The datasets analyzed are available with the corresponding author on reasonable request. 


\section{Ethics approval and consent to participate}

The study was approved by the University of Antioquia Bioethics Committee of the Institute of Medical Research and the Ethics Committee of the Hospitals; informed consent was obtained from all participants included in the study.

\section{Consent for publication}

All authors agree to the publication of the manuscript.

\section{Competing interests}

The authors do not have any conflicts of interest.

\section{Author details}

'GRAEPIC —Clinical Epidemiology Academic Group (Grupo Académico de Epidemiología Clínica), the University of Antioquia, Medellín, Colombia. ${ }^{2}$ University of Antioquia, Medellín, Colombia. ${ }^{3}$ Department of Internal Medicine, University of Antioquia, Medellín, Colombia. ${ }^{4}$ Hospital San Vicente Fundación, Medellín, Colombia.

Received: 13 February 2020 Accepted: 15 April 2020

Published online: 22 April 2020

\section{References}

1. Fleischmann C, Scherag A, Adhikari NK, Hartog CS, Tsaganos T, Schlattmann $P$, et al. Assessment of global incidence and mortality of hospital-treated sepsis. Current Estimates and Limitations. Am J Respir Crit Care Med. 2016; 193:259-72.

2. Dellinger RP, Carlet JM, Masur H, Gerlach H, Calandra T, Cohen J, et al. Surviving Sepsis Campaign guidelines for management of severe sepsis and septic shock. Crit Care Med. 2004;32:858-73.

3. Damiani E, Donati A, Serafini G, Rinaldi L, Adrario E, Pelaia P, et al. Effect of performance improvement programs on compliance with sepsis bundles and mortality: a systematic review and meta-analysis of observational studies. PLoS One. 2015:10:e0125827.

4. Marik PE, Farkas JD, Spiegel R, Weingart S, Aberegg S, Beck-Esmay J, et al. POINT: should the Surviving Sepsis Campaign guidelines be retired? Yes. Chest. 2019;155:12-4.

5. Levy MM, Rhodes A, Evans LE, Antonelli M, Bailey H, Kesecioglu J, et al. COUNTERPOINT: should the Surviving Sepsis Campaign guidelines be retired? No. Chest. 2019:155:14-7.

6. Sterling SA, Miller WR, Pryor J, Puskarich MA, Jones AE. The impact of timing of antibiotics on outcomes in severe sepsis and septic shock: a systematic review and meta-analysis. Crit Care Med. 2015:43:1907-15.

7. Hranjec T, Rosenberger L, Swenson B, Metzger R, Flohr T, Politano A, et al. Aggressive versus conservative initiation of antimicrobial treatment in critically ill surgical patients with suspected intensive-care-unit-acquired infection: a quasi-experimental, before and after observational cohort study. Lancet Infect Dis. 2012;12:774-80.

8. Rosenbaum PR, Rubin DB. The central role of the propensity score in observational studies for causal effects. Biometrika. 1983;70:41-55.

9. D'Agostino RB Jr, D'Agostino RB Sr. Estimating treatment effects using observational data. JAMA. 2007;297:314-6.

10. Londono J, Nino C, Archila A, Valencia M, Cardenas D, Perdomo M, et al. Antibiotics has more impact on mortality than other early goal-directed therapy components in patients with sepsis: an instrumental variable analysis. J Crit Care. 2018;48:191-7.

11. Horan TC, Andrus M, Dudeck MA. CDC/NHSN surveillance definition of health care-associated infection and criteria for specific types of infections in the acute care setting. Am J Infect Control. 2008;36:309-32.

12. Rivers E, Nguyen B, Havstad S, Ressler J, Muzzin A, Knoblich B, et al. Early goal-directed therapy in the treatment of severe sepsis and septic shock. $\mathrm{N}$ Engl J Med. 2001;345:1368-77.

13. Charlson ME, Pompei P, Ales KL, Mackenzie CR. A new method of classifying prognostic comorbidity in longitudinal studies: development and validation. J Chronic Dis. 1987:40:373-83.

14. Vincent JL, Moreno R, Takala J, Willatts S, De Mendonca A, Bruining H, et al. The SOFA (Sepsis-related Organ Failure Assessment) score to describe organ dysfunction/failure. On behalf of the Working Group on Sepsis-Related Problems of the European Society of Intensive Care Medicine. Intensive Care Med. 1996;22:707-10.
15. Knaus WA, Draper EA, Wagner DP, Zimmerman JE. APACHE II: a severity of disease classification system. Crit Care Med. 1985;13:818-29.

16. Agoritsas T, Merglen A, Shah ND, O'Donnell M, Guyatt GH. Adjusted analyses in studies addressing therapy and harm: users' guides to the medical literature. JAMA. 2017;317:748-59.

17. Stuart EA. Matching methods for causal inference: a review and a look forward. Stat Sci. 2010;25:1-21.

18. Singer M, Deutschman CS, Seymour CW, Shankar-Hari M, Annane D, Bauer M, et al. The Third International Consensus Definitions for Sepsis and Septic Shock (Sepsis-3). JAMA. 2016;315:801-10.

19. von Elm E, Altman DG, Egger M, Pocock SJ, Gotzsche PC, Vandenbroucke JP. The Strengthening the Reporting of Observational Studies in Epidemiology (STROBE) statement: guidelines for reporting observational studies. J Clin Epidemiol. 2008;61:344-9.

20. Levy MM, Evans LE, Rhodes A. The Surviving Sepsis Campaign bundle: 2018 update. Intensive Care Med. 2018;44:925-8.

21. Liu VX, Fielding-Singh V, Greene JD, Baker JM, Iwashyna TJ, Bhattacharya J, et al. The timing of early antibiotics and hospital mortality in sepsis. Am J Respir Crit Care Med. 2017;196:856-63.

22. Seymour CW, Gesten F, Prescott HC, Friedrich ME, Iwashyna TJ, Phillips GS, et al. Time to treatment and mortality during mandated emergency care for sepsis. N Engl J Med. 2017:376:2235-44.

23. Kumar A, Zarychanski R, Light B, Parrillo J, Maki D, Simon D, et al. Early combination antibiotic therapy yields improved survival compared with monotherapy in septic shock: a propensity-matched analysis. Crit Care Med. 2010:38:1773-85.

24. Ferrer R, Martin-Loeches I, Phillips G, Osborn TM, Townsend S, Dellinger RP, et al. Empiric antibiotic treatment reduces mortality in severe sepsis and septic shock from the first hour: results from a guideline-based performance improvement program. Crit Care Med. 2014;42:1749-55.

25. Pruinelli L, Westra BL, Yadav P, Hoff A, Steinbach M, Kumar V, et al. Delay within the 3-hour Surviving Sepsis Campaign guideline on mortality for patients with severe sepsis and septic shock. Crit Care Med. 2018;46:500-5.

26. Sherwin R, Winters ME, Vilke GM, Wardi G. Does early and appropriate antibiotic administration improve mortality in emergency department patients with severe sepsis or septic shock? J Emerg Med. 2017;53:588-95.

27. Singer M. Antibiotics for Sepsis: Does each hour really count, or is it incestuous amplification? Am J Respir Crit Care Med. 2017;196:800-2.

28. de Groot B, Ansems A, Gerling DH, Rijpsma D, van Amstel P, Linzel D, et al. The association between time to antibiotics and relevant clinical outcomes in emergency department patients with various stages of sepsis: a prospective multi-center study. Crit Care. 2015;19:194.

29. Abe T, Kushimoto S, Tokuda Y, Phillips GS, Rhodes A, Sugiyama T, et al. Implementation of earlier antibiotic administration in patients with severe sepsis and septic shock in Japan: a descriptive analysis of a prospective observational study. Crit Care. 2019;23:360.

30. Alam N, Oskam E, Stassen PM, Exter PV, van de Ven PM, Haak HR, et al. Prehospital antibiotics in the ambulance for sepsis: a multicentre, open label, randomised trial. Lancet Respir Med. 2018;6:40-50.

31. Vattanavanit $\mathrm{V}$, Buppodom $\mathrm{T}$, Khwannimit B. Timing of antibiotic administration and lactate measurement in septic shock patients: a comparison between hospital wards and the emergency department. Infect Drug Resist. 2018;11:125-32.

32. Puskarich MA, Trzeciak S, Shapiro NI, Arnold RC, Horton JM, Studnek JR, et al. Association between timing of antibiotic administration and mortality from septic shock in patients treated with a quantitative resuscitation protocol. Crit Care Med. 2011;39:2066-71.

33. Kumar A, Roberts D, Wood KE, Light B, Parrillo JE, Sharma S, et al. Duration of hypotension before initiation of effective antimicrobial therapy is the critical determinant of survival in human septic shock. Crit Care Med. 2006;34:1589-96.

34. Peltan ID, Brown SM, Bledsoe JR, Sorensen J, Samore MH, Allen TL, et al. ED doorto-antibiotic time and long-term mortality in sepsis. Chest. 2019;155:938-46.

35. Aslam B, Wang W, Arshad MI, Khurshid M, Muzammil S, Rasool MH, et al. Antibiotic resistance: a rundown of a global crisis. Infect Drug Resist. 2018; 11:1645-58.

36. Klompas M, Calandra T, Singer M. Antibiotics for sepsis-finding the equilibrium. JAMA. 2018;320:1433-4.

\section{Publisher's Note}

Springer Nature remains neutral with regard to jurisdictional claims in published maps and institutional affiliations. 